\section{Periodontal probing made simple}

Streamline periodontal examinations with the advanced Florida Probe, which is available to purchase as a traditional kit, or as the intuitive Voice Works system for ultimate ease-of-use.

With highly efficient computerised probing at your fingertips, the Florida Probe provides consistent precision $(0.2 \mathrm{~mm})$ and

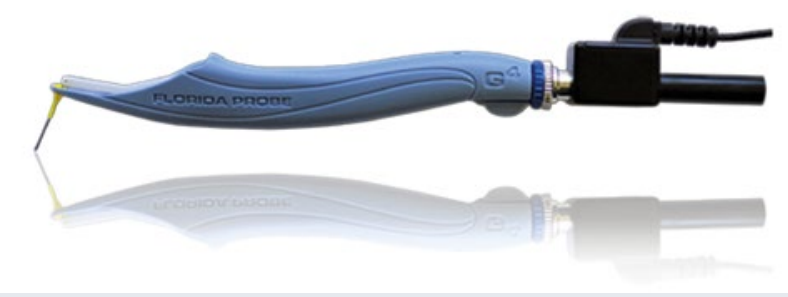

force (15 g), enabling practitioners to achieve highly accurate results.

Clinicians can feel confident knowing they are able to operate alone using the Florida Probe's software, which offers post-examination hand-outs that can be used to help improve patient-practitioner communication, and increase treatment acceptance rates.

More information is available by calling Clark Dental on 01268733 146, emailing info@clarkdental.co.uk or visiting www.clarkdental.co.uk.

\title{
Next step CAD/CAM restorations
}

Unlike most other CAD/CAM blocs, BRILLIANT Crios has a multimodal composition of dental glass and amorphous silica, which along with a reinforcing resin matrix make it ideal for permanent single-tooth restorations.

By using this next generation composition, dentists can benefit from high flexural strength and low modulus of elasticity that is similar to dentine, which offers a shock absorbing ability and pleasant bite.
Dr Bertie Napier, owner of Dentistry19 in Hertfordshire, says: 'It helps too that the BRILLIANT Crios adapts nicely to the surrounding natural tooth and is very easy to polish to a high, long-lasting lustre. I would definitely recommend COLTENE's reinforced composite bloc to others.'

Find out more by visiting www. coltene.com emailinginfo.uk@ coltene.com or calling 01444235486.

\section{Innovation for intraoral scanning}

Sometimes in technological developments, there are seminal moments that can change the direction of travel for a particular service or industry - and so it was on 1 February 2019, when more than 300 selected dentists, technicians and media from all over Europe, witnessed the launch of Primescan by Dentsply Sirona.

The launch venue was the Klassikstadt in Frankfurt, Germany, for the launch event of what the company describes as a project that has been more than five years in the making and the culmination of thousands of hours of research, development, testing and refinement.

It is a platform from which dentistry can launch itself into a new era of clinical excellence and patient satisfaction.

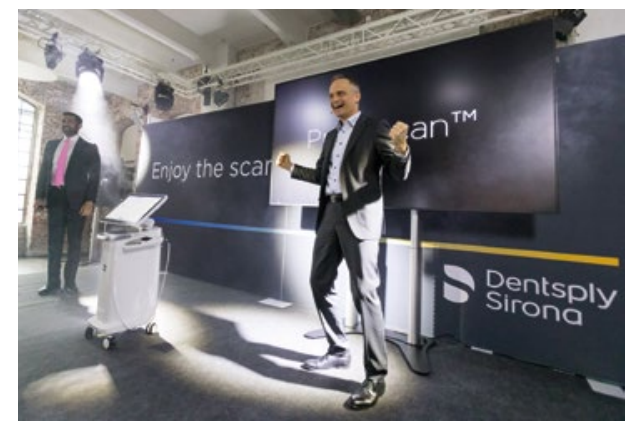

bringing CAD/CAM into the mainstream, he said.

Now with the launch of Primescan, Dentsply Sirona can refute these accusations even further, as it lays down the gauntlet to other manufacturers by setting a standard way above anything else currently available.

Primescan's unique technology means the user can capture more high-resolution data more quickly, scanning a whole jaw in less than 30 seconds. During the scanning process the dynamic lens detects more than one million data points per second, whilst intelligent processing compresses the high volume of data, enabling models to be calculated faster.

Primescan consolidates more than 50,000 images per second, creating photorealistic images of a quality that has never been achieved before.

The event host Dr Sameer Puri, Director of CAD/CAM at Spear and owner of the website www.cerecdoctors.com denied suggestions that CEREC had lost ground against its competitors and been 'left behind' in the new era of intraoral scanning.

Such murmurings, confirmed Dr Puri, were completely unfounded. In 2018, more than seven million scans were completed using CEREC Omnicam. When combined with Dentsply Sirona's material and milling capabilities it created a powerful system that started the journey of
It is also flexible and intuitive. It accommodates faster access to the tooth surface without the need for significant tilting of the camera head, and the increased field of vision enables larger areas to be scanned with fewer sweeps.

To find out more about the Dentsply Sirona range of digital solutions, visit www.dentsplysirona.com or call 01932838355. 